\title{
Pharyngocutaneous fistula as an alternative access route for inserting a percutaneous endoscopic gastrostomy tube in head and neck cancer patients
}

\section{다)(1) $(-)=$}

\author{
Authors \\ Louise Deluiz Verdolin Di Palma, Gustavo Francisco de Souza e Mello, Cindy Lis Granados, Ricardo Dardengo Glória, \\ Caroline Sauter Dalbem, Rolantre Lopes da Cruz, Ana Carolina Maron Ayres, Renata Sofia Camara Lisboa, Alexandre \\ Dias Pelosi, Maria Aparecida Ferreira, Gilberto Reynaldo Mansur, Simone Guaraldi da Silva, Theresa Christina Damian \\ Ribeiro, Fernando Luiz Dias
}

Institution

Department of Digestive Endoscopy, Cancer Hospital I, Brazilian National Cancer Institute, Rio de Janeiro, Brazil

submitted: 16.8.2016

accepted after revision: 2.3 .2017

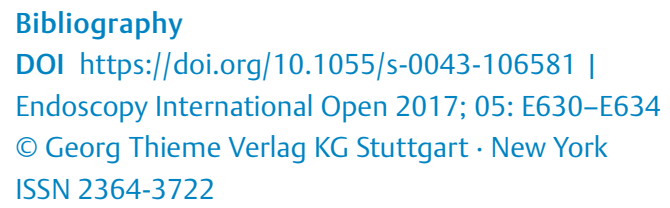

Corresponding author

Gustavo Francisco de Souza e Mello, MD, PhD, Department of Digestive Endoscopy, Cancer Hospital I, Brazilian National Cancer Institute, Praça Cruz Vermelha 23, Rio de Janeiro RJ, CEP 20230-130

gmello@inca.gov.br

gfsmello@gmail.com

\section{ABSTRACT}

Background and study aims Performing a percutaneous endoscopic gastrostomy (PEG) in head and neck cancer (HNC) patients can be challenging because of the presence of trismus, pharyngeal obstruction by tumor, and pharyn- goesophageal strictures or fistula. Pharyngocutaneous fistula (PCF) is a major postoperative concern in patients submitted to total laryngectomy ( $\mathrm{TL})$. In the medical literature to date, the cervical fistula has been used as an access to PEG in only four reports. The aim of this study was to evaluate the safety of cervical fistula for insertion of a PEG tube. Patients and methods Retrospective study at a single tertiary referral center, regarding the technical feasibility, safety and outcomes of a PEG tube introduced by a cervical fistula in HNC patients with obstructive lesions of the oropharynx.

Results The procedure was technically successful in all 21 patients. A PEG tube was used for a minimum of 1 month and a maximum of 120 months. Twelve patients died while using the PEG tube, 8 had it taken out because it was no longer needed, and only 1 had the tube still in use. Adverse events occurred in 8 patients: granuloma (19\%), dermatitis (9.5\%), accidental late removal of the tube (9.5\%), periprocedural gastric wall hematoma $(9.5 \%)$, peristomal wound infection $(4.7 \%)$, buried bumper syndrome $(4.7 \%)$, and traumatic gastric ulcer (4.7\%).

Conclusion A postoperative cervical fistula can successfully work as a reliable and safe access for a PEG tube procedure in HNC patients, avoiding unnecessary surgery and reducing costs.

\section{Introduction}

Nutritional support is a critical point in the care of patients with advanced cancer. In general, patients with malignancies suffer malnutrition from tumor burden and/or anorexia. Head and neck cancer (HNC) patients have additional problems because swallowing disorders are caused by pharyngeal strictures, fistula, and damage to the pharyngeal musculature resulting from the tumor, radiation therapy, or surgical procedure. Nevertheless, the gastrointestinal tract in these individuals usually is in- tact, making them fit for enteral nutrition. In these patients, nourishment can be administered through nasoenteric tubes or gastrostomy tubes placed by endoscopy, radioscopy or laparotomy $[1,2]$.

In 1980, Gauderer et al. first described insertion of a percutaneous gastrostomy tube under local anesthesia using endoscopy, in a child [3]. A percutaneous endoscopic gastrostomy (PEG) tube has rapidly become the most widely used method for long-term feeding, because it is safer and more cost-effec- 
tive than surgically placed gastrostomies, with a low rates of procedure-related mortality and complications [4, 5].

Considerations for performing a PEG in HNC patients include dealing with the presence of trismus, pharyngeal obstruction caused by tumor mass, pharyngoesophageal strictures or fistula, difficult airway, and the timing of PEG with respect to surgery, radiation, and chemotherapy for the oropharyngeal malignancy $[1,6]$.

Because of these difficulties, endoscopists have to be resourceful and find different ways to bypass the technical and anatomic limitations. Regarding the strictures, maneuvers that allow PEG tube placement in these patients include the selection of a small-caliber endoscope and pharyngoesophageal dilatation [1]. In different reports, upper aerodigestive tract obstruction accounted for $20 \%$ to $75 \%$ of all PEG tube placement failures in HNC patients [1,7]. In some clinical scenarios with surgical HNC patients, if an endoscopist is unable to bypass a local stricture or a trismus, a postoperative cervical fistula (frequent treatment sequelae in these patients) or an esophagostomy can be used to introduce the endoscope.

Pharyngocutaneous fistula (PCF) is a major post-treatment concern in patients who undergo total laryngectomy $(\mathrm{TL})$. The reported incidence of PCF following TL for laryngeal cancer ranges from $2.6 \%$ to $65.5 \%$ [8]. The fistula may be secondary to impaired wound healing, persistent distal obstruction to salivary flow, technical dificulties during pharyngoplasty or local tissue ischemic necrosis, which general occurs within the first few postoperative days $[9,10]$.

The first time an esophagocutaneous fistula was used for access to insert a PEG tube was reported by Hunter et al. in 1989 [1], when they described 1 case among 50 successful PEGs in HNC patients. Since then, only a few reports have been described in the medical literature. Our aim was to report our experience with use of a postoperative cervical fistula as an alternative access route for PEG tube placement in HNC patients with obstructive lesions of the oropharyngeal region.

\section{Patients and methods}

\section{Study design}

Retrospective review of prospectively collected clinical data, presented as a case series of consecutive HNC patients undergoing PEG done through a post-treatment cervical fistula or stoma, conducted at the Department of Digestive Endoscopy of the Brazilian National Cancer Institute (INCA), a federal government tertiary referral center, at Rio de Janeiro, Brazil.

Clinical and demographic data on the patients, adverse events (AEs), and patient outcomes were recorded in an Excel spreadsheet (Microsoft Corp., Redmond, Washington, United States) during long-term follow-up.

This study protocol was approved by the INCA Institutional Review Board (CEP/INCA Number 1706). Informed consent was obtained from all patients.

\section{Study population}

From January 2002 to December 2014, 1305 PEG procedures were done in HNC patients at our institution. Of them, 21 PEG procedures were performed in the same number of adult HNC patients with obstructive lesions of the oral cavity or hypopharynx by inserting the endoscope and PEG tube directly through a cervical fistula or stoma.

\section{Operative technique}

Prophylactic intravenous cephazolin $(2 \mathrm{~g})$ was given to all patients 30 minutes before the procedure. Under pulse oximetry continuous monitoring and conscious sedation with intravenous midazolam and meperidine, a standard-diameter or pediatric gastroesophagoscope was inserted through the cervical (pharyngo or esophagocutaneous) fistula, and the upper gastrointestinal tract was thoroughly examined via endoscopy. PEG was performed using the "pull" method (Gauderer-Ponsky technique) using commercially available kits (PEG 24-Pull-S, Cook Medical, Winston-Salem, North Carolina, United States; MIC PEG 24Fr, Ballard Medical Products, Draper, Utah, United States; and EndoVive PEG 24 Fr, Boston Scientific Corporation, Natick, Massachusetts, United States).

\section{Follow-up}

Following the institutional post-PEG protocol, patients returned for regular visits at scheduled intervals (on post-procedure days 2 and 7 , and then monthly) during the period of PEG use, until the tube was removed or the patient died.

\section{Definition of adverse events}

AEs were considered minor when they required simple or ambulatory care (late dislodged tube, peristomal leak, wound infection, granuloma formation, minor wound bleeding or small hematoma). Major AEs included peritonitis, buried bumper syndrome (BBS), septicemia, aspiration pneumonia, hemorrhage, gastric perforation, gastrocolocutaneous fistula and any AEs requiring a repeat procedure, surgical or endoscopic treatment, or blood transfusion [11].

\section{Results}

\section{Results are summarized in $>$ Table $\mathbf{1}$.}

A standard-diameter or pediatric gastroesophagoscope was passed through a cervical fistula or stoma and a pull-technique PEG was performed in a total of $21 \mathrm{HNC}$ patients ( 3 female and 18 male). The age of patients varied from 34 to 84 years (mean 59 years).

Eighteen (85.7\%) patients had laryngeal cancer, 2 (9.5\%) had oropharyngeal cancer, and 1 (4.7\%) had hypopharyngeal cancer. All of them had undergone a radical surgical procedure (2 had a combined mandibulectomy and neck dissection operation [COMMANDO surgery], and 19 had a total laryngectomy). Four $(19 \%)$ of those patients had complementary treatment with chemotherapy and radiation therapy, and 9 (42.8\%) had radiation therapy alone. 


\begin{tabular}{|l|c|}
\hline \begin{tabular}{l} 
- Table 1 Patient characteristics. \\
\hline HNC patients, no. (\%)
\end{tabular} & $21(100)$ \\
\hline Age, median (IQR), y & $59(34-84)$ \\
\hline Gender, no. (\%) & $18(85.7)$ \\
\hline - Male & $3(14.3)$ \\
\hline - Female & \\
\hline Tumor location, no. (\%) & $18(85.7)$ \\
\hline - Larynx & $2(9.5)$ \\
\hline - Oropharynx & $1(4.8)$ \\
\hline - Hypopharynx & \\
\hline Tumor treatment, no. (\%) & $9(42.7)$ \\
\hline - Surgery + RXT & $7(33.3)$ \\
\hline - Surgery & $4(19.0)$ \\
\hline - Surgery + RXT + CHT
\end{tabular}

\begin{tabular}{|l|c|}
\hline \multicolumn{2}{|l|}{ PEG procedure setting, no. (\%) } \\
\hline - Inpatient & $13(61.9)$ \\
\hline - Outpatient (ambulatory) & $8(38.1)$ \\
\hline PEG procedures with complication, no. (\%) $)^{1}$ & $8(38.1)$ \\
\hline - Major & $1(4.8)$ \\
\hline - Minor & $8(38.1)$ \\
\hline
\end{tabular}

\begin{tabular}{|l|l|}
\hline PEG adverse event, no. $(\%)^{1}$ & \\
\hline - Granulation tissue & $4(19.0)$ \\
\hline - Dermatitis & $2(9.5)$ \\
\hline - PEG tube placement & $2(9.5)$ \\
\hline - Gastric hematoma & $2(9.5)$ \\
\hline - Stomal infection & $1(4.8)$ \\
\hline - Traumatic gastric ulcer & $1(4.8)$ \\
\hline - BBS & $1(4.8)$ \\
\hline
\end{tabular}

\begin{tabular}{|l|l|}
\hline Follow-up, median (IQR), m & $17.9(1-120)$ \\
\hline
\end{tabular}

Outcome, no. (\%)

\begin{tabular}{|l|l} 
- Death (disease progression) & $12(57.1)$
\end{tabular}

- PEG removed 8(38.1)

- PEG in use $1(4.8)$

HNC, head and neck cancer; no, number; \%, percent; IQR, interquartile range; y, years; RXT, radiotherapy; $\mathrm{CHT}$, chemotherapy; PEG, percutaneous endoscopic gastrostomy; BBS, buried bumper syndrome; $m$, months;

${ }^{1} 8$ patients with a total of 13 adverse events

PEG was performed as an outpatient procedure (ambulatory setting, without hospitalization) in $8(38.1 \%)$ of 21 patients.

Preprocedure endoscopic dilation of a fibrotic and narrowed esophageal opening (with a Savary bougie or a hydrostatic bal-
Ion) was necessary for $3(14.3 \%)$ patients, to allow advancement of the endoscope.

Eight patients $(38.1 \%)$ presented with PEG complications, with more than one event per patient in 4 cases ( 1 patient with 3 different complications and 3 patients with 2 complications each), for a total of 13 AEs: granuloma formation (4 cases; $19 \%$ ), dermatitis ( 2 cases; $9.5 \%$ ), accidental late removal of the tube (2 cases; $9.5 \%$ ), periprocedural gastric wall hematoma (2 cases; $9.5 \%$ ), peristomal wound infection (1 case; $4.8 \%$ ), BBS (1 case; $4.8 \%$ ), traumatic gastric ulcer (1 case; $4.8 \%$ ). The rate of major AEs was $4.8 \%$ (1 patient) and minor AEs was $38.1 \%$ (8 patients). There were no fatalities related to the PEG procedure.

In the follow-up, a PEG tube was used for a minimum of 1 month and a maximum of 120 months (mean of 17.9 months). Of all the patients, 12 (57.1\%) died while using the PEG tube, 8 $(38.1 \%)$ had the PEG tube taken out because it was no longer needed, and only 1 (4.8\%) had the tube still in use.

\section{Discussion}

Numerous techniques are currently available for inserting a PEG tube in difficult HNC patients $[12,13]$. The route for introducing the endoscope into the stomach is one of the crucial points of the procedure. In most cases, peroral gastroscopy and pulltechnique PEG placement are easy and safe. However, in the presence of severe trismus, tumor obstruction, or post-treatment anatomic changes, the procedure may become impractical. Introducer PEG technique by transnasal approach with ultrathin endoscopes, rigid laryngoscopic guidance, radiologic percutaneous gastrostomy (RPG), transmaxillary approach, intraoperative procedures (surgically opened pharynx region), and open surgical gastrostomy must be considered to insert a G-tube successfully in these difficult cases $[12,13]$. To avoid a surgical procedure, an existing cervical fistula or ostomy may be used to provide access to the PEG tube, if the procedure cannot be performed transorally $[1,14,15]$.

For the $21 \mathrm{HNC}$ patients included in this case series, there were 2 main indications for the introduction of the endoscope directly through a cervical fistula or stoma. The first (and more frequent indication) was the finding of a tumoral or cicatricial obstruction of the oral cavity or hypopharynx region that precluded tube progression. The second reason was the presence of an extensive anterior cervical wall defect that resulted in complete exteriorization of the endoscope tube after the transposition of the hypopharynx ( $\triangleright$ Fig. 1). In the latter situation, even it was possible to advance the tube, introduction of the endoscope through the mouth would result in an unnecessary maneuver and pose an elevated risk of contamination of the PEG tube, which could result in a higher rate of peristomal infection. In the majority of cases, the two situations were present simultaneously.

Until today, only 4 reports in the medical literature have described use of cervical access (by a preexisting fistula or stoma) to perform a PEG in HNC patients [1, 14-16].

Hunter et al. reported the first description of PEG tube insertion through a cervical fistula in 1989 [1]. At that time, they presented the experience of performing PEG in 50 patients 

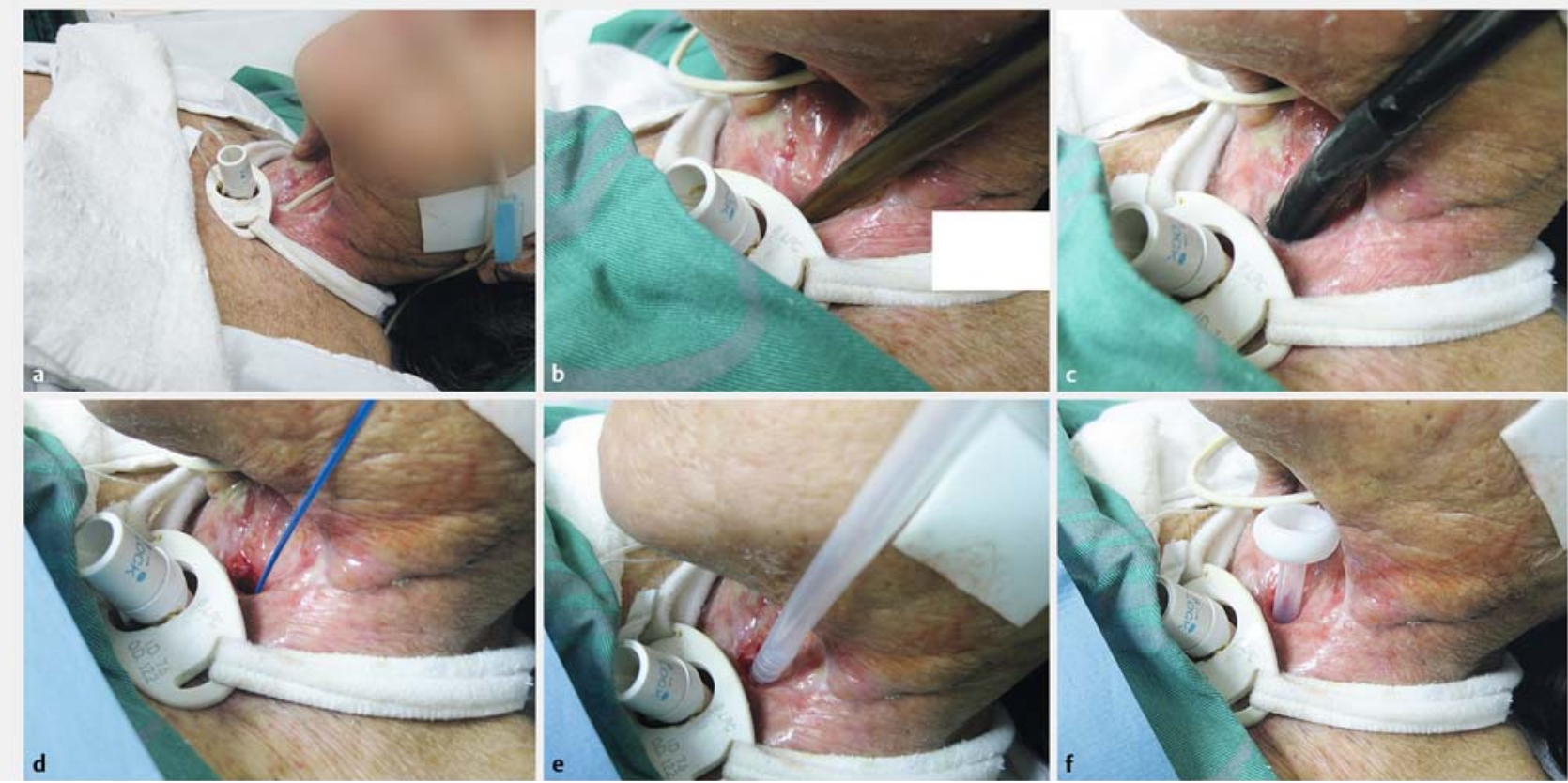

- Fig. 1 Alternative access route for inserting a PEG tube using the "pull" method (Gauderer-Ponsky technique) a External aspect of the anterior cervical wall defect. A nasoenteric tube is seen across the exposed posterior wall of the pharyngeal region. A tracheostomy tube is in place. b Dilation of the narrowed and fibrotic esophageal opening with a Savary bougie.c Endoscope introduced in the esophagus. $\mathbf{d}$ The guide-wire is pulled out of the esophagus and connected to the PEG tube. e PEG tube insertion through the cervical opening. $\mathbf{f}$ PEG tube internal bumper advancement.

with advanced oropharyngeal cancer, using a pull-through PEG technique. In one of the patients they used an esophagocutaneous fistula as an access via to inserting the PEG tube.

In 2001, Lujber et al. described a 59-year-old patient with a squamous cell carcinoma of the left piriform sinus who had radiation and surgical treatment [14]. Two weeks after the operation, formation of fistula began in the neck. Since the oral route could not be used because of extensive irradiationinduced fibrosis and the postoperative narrowing lumen of the remaining hypopharynx, the fistula seemed the best way to access the stomach. Unlike our patients, who underwent PEG under conscious sedation, the procedure in Lujber et al.'s study was done under general anesthesia. Insertion of an $18 \mathrm{Fr}$ PEG tube insertion through the orifice of the fistulae was successful. No major or minor AEs occurred during or after the procedure. The PEG tube was removed 4 months later. The patient had no tumor recurrence or other problems during the 24-month follow-up period.

In 2006, Lujber described 3 other cases of PEG tube insertion through a cervical fistula [15]. In all cases, the pull-type PEG technique was used. Only 1 patient had the procedure done under general anesthesia, the other 2 had local anesthesia. No immediate or long-term AEs were noted.

In a report of PEG as an outpatient procedure in HNC patients, Mello et al. used cervical fistula and pharyngoesophagostomies as the access route for introducing the endoscope and the PEG tube in 6 (4.6\%) of a total of 136 patients [16].

In all mentioned cases above, a pharyngoesophagocutaneous fistula was used to access the stomach lumen, and a pull-technique PEG was successfully performed. As seen, this procedure can be done using general anesthesia or intravenous conscious sedation.

All of our procedures were safely done with pull-type PEG kits (Gauderer-Ponsky method), at the endoscopy room and under conscious intravenous sedation (avoiding unnecessary general anesthesia and mechanical ventilation). In almost $40 \%$ of cases, PEG was performed as an outpatient procedure, in the ambulatory setting. At our institution, both introducer-type PEG technique (Russell method) and RPG were only regularly available after 2012.

Lujber et al. and Lujber had no AEs in any of their 4 procedures $[14,15]$. Hunter et al. had AEs events classified as minor without further specifications [1]. In our report, only one major AE (a buried bumper syndrome) was seen, all other AEs were classified as minor. No patient died from the PEG procedure. Some authors reported a total AE rate of $37 \%$ to $40 \%$ in HNC patients submitted to PEG [17-20]. A systematic review of PEG in HNC patients reported minor AEs in 171 (13.3\%) of 1281 procedures and major AEs in 60 (4.6\%) [11]. Mortality occurred in 10 $(0.8 \%)$ patients. Compared to PEG literature in HNC patients, our AEs occurred at the expected frequency, regardless of the relationship to or influence of introduction of the endoscope and/or PEG tube through the cervical fistula. 


\section{Conclusion}

When dealing with HNC patients, the PEG procedure can be a challenge due to obstructive lesions or trismus. This report registers that a pharyngoesophagocutaneous fistula, a frequent post-treatment $A E$ in these patients, can successfully be used as a reliable and safe access route to PEG tube introduction, avoiding unnecessary surgery and reducing costs.

Competing interests

None

\section{References}

[1] Hunter JG, Lauretano L, Shellito PC. Percutaneous endoscopic gastrostomy in head and neck cancer patients. Ann Surg 1989; 210: 42 46

[2] Pearce CB, Duncan HD. Enteral feeding. Nasogastric, nasojejunal, percutaneous endoscopic gastrostomy, or jejunostomy: its indications and limitations. Postgrad Med J 2002; 78: $198-204$

[3] Gauderer MWL, Ponsky JL, Izant RJ Jr. Gastrostomy without laparotomy: a percutaneous endoscopic technique. J Pediatr Surg 1980; 15: $872-875$

[4] Moran B], Taylor MB, Johnson CD. Percutaneous endoscopic gastrostomy. Br J Surg 1990; 77: 858-862

[5] Rustom IK, Jebreel A, Tayyab M et al. Percutaneous endoscopic, radiological and surgical gastrostomy tubes: a comparison study in head and neck cancer patients. J Laryngol Otol 2006; 120: 463-466

[6] Nevah MI, Lamberth JR, Dekovich AA. Transnasal PEG tube placement in patients with head and neck cancer. Gastrointest Endosc 2014; 79: $599-604$

[7] Miller RE, Kummer BA, Kotler DP et al. Percutaneous endoscopic gastrostomy: procedure of choice. Ann Surg 1986; 204: 543 - 545
[8] Paydarfar J, Birkmeyer NJ. Complications in head and neck surgery: a meta-analysis of postlaryngectomy pharyngocutaneous fistula. Arch Otolaryngol Head Neck Surg 2006; 132: 67-72

[9] Genden EM, Rinaldo A, Shaha AR et al. Pharyngocutaneous fistula following laryngectomy. Acta Otolaryngol 2004; 124: 117-120

[10] Galli ], Corso ED, Volante M et al. Postlaryngectomy pharyngocutaneous fistula: incidence, predisposing factors, and therapy. Otolaryngology-Head and Neck Surg 2005; 133: 689-694

[11] Grant DG, Bradley PT, Pothier DD et al. Complications following gastrostomy tube insertion in patients with head and neck cancer: a prospective multi-institution study, systematic review and meta-analysis. Clin Otolaryngol 2009; 34: $103-112$

[12] Taller A, Horvath E, lliás L et al. Technical modifications for improving the success rate of PEG tube placement in patients with head and neck cancer. Gastrointest Endosc 2001; 54: 633-636

[13] Grier JF, Goldman DE, Gholson CF. Transmaxillary percutaneous endoscopic gastrostomy. Gastrointest Endosc 1994; 40: 778 - 779

[14] Lujber L, Fábián G, Pytel J. Inserting a percutaneous endoscopic gastrostomy tube via a cervical fistula formed after major surgery on a patient with a head and neck tumor. Surg Laparosc Endosc Percutaneous Techn 2001; 11: 327-329

[15] Lujber L. Placement of a percutaneous endoscopic gastrostomy feeding tube via a cervical pharyngocutaneous fistula. Endoscopy 2006; 38: $\mathrm{E} 57$

[16] Mello GFS, Lukashok HP, Meine GC et al. Outpatient percutaneous endoscopic gastrostomy in selected head and neck cancer patients. Surg Endosc 2009; 23: 1487 - 1493

[17] Ehrsson YT, Langius-Eklof A, Bark T et al. Percutaneous endoscopic gastrostomy (PEG) - a long-term follow-up study in head and neck cancer patients. Clin Otolaryngol 2004; 29: 740-746

[18] Raynor EM, Williams MF, Martindale RG et al. Timing of percutaneous endoscopic gastrostomy tube placement in head and neck cancer patients. Otolaryngol Head Neck Surg 1999; 120: 479-482

[19] Walton GM. Complications of percutaneous endoscopic gastrostomy in patients with head and neck cancer - an anatysis of 42 consecutive patients. Ann R Coll Surg Engl 1999; 81: 272-276

[20] Ruston IK, Jebreel A, Tayyab M et al. Percutaneous endoscopic, radiological and surgical gastrostomy tubes: a comparison study in head and neck cancer patients. J Laryngol Otology 2006; 120: $463-466$ 